\title{
ON THE NUMBER OF SETS CONJUGATE TO A MATRIX WITH LINEAR ELEMENTARY DIVISORS*
}

\author{
H. S. THURSTON
}

1. Introduction. The determination of sets of conjugates to a given matrix $M$ with respect to its minimum equation was first discussed by E. S. Sokolnikoff in 1933. $†$ In two papers published more recently, one $\ddagger$ by E. T. Browne and one§ by the writer, the subject has received further consideration, and, in particular, attention has been paid to the case in which $M$ has only linear elementary divisors. In the former of these two papers, conjugates were obtained by employing the principal idempotent elements of $M$. The second paper considered only conjugates of $M$ in a ring $R(A)$ where $A$ had simple latent roots, and made use of the principal idempotent elements of $A$.

Since each principal idempotent element of $M$ is a set of one or more of its partial idempotent elements, there will be, in general, more sets of conjugates obtained by using the latter than by using the former. This fact was stated by both Sokolnikoff and Browne. Not all of these conjugates are polynomials in $M$. The writer exhibited sets of matrices in the ring $R(A)$ conjugate to $M$ but not expressible as polynomials in $M$. These are accounted for by the fact that the principal idempotent elements of $A$ are the partial idempotent elements of $M$.

It is the purpose of this paper to show that there exists a nonderogatory matrix $A$ (by no means unique), such that any matrix $M$ can be expressed as a polynomial in $A$. Then for the restricted case under consideration, the exact numbers of conjugate sets in the rings $R(A)$ and $R(M)$ can be determined.

2. Determination of the matrix $A$. Let $M$ be any square matrix of order $n$. Then there exists a nonsingular matrix $T$ such that $T^{-1} M T=C$, where $C$ is in the Jordan canonical form. Let $B$ be any matrix in Jordan canonical form whose nonoverlapping blocks are of the same order as those in $C$, and having no one latent root occurring in two such blocks. It is easily shown that $C$ is an integral, rational function of $B$, say $f(B)$. Then, if $A=T B T^{-1}$, it follows that

\footnotetext{
* Presented to the Society, September 6, 1938.

$\dagger$ E. S. Sokolnikoff, American Journal of Mathematics, vol. 55 (1933), pp. 167180.

$\ddagger$ E. T. Browne, ibid, vol. 59 (1937), pp. 845-868.

$\S$ H. S. Thurston, this Bulletin, vol. 44 (1938), pp. 258-261.
} 


$$
M=T C T^{-1}=T \cdot f(B) \cdot T^{-1}=f\left(T B T^{-1}\right)=f(A) .
$$

Hence we have the following theorem:

THEOREM 1. If $M$ is any square matrix, there can be found a nonderogatory matrix $A$ such that $M$ is expressible as a polynomial in $A$.

3. Sets of conjugates to $M$. We shall now restrict our attention to the case where $M$ has only linear elementary divisors. The matrix $A$ determined in the above theorem then has only simple latent roots. If the characteristic function of $M$ is $\prod_{i=1}^{r}\left(\lambda-\alpha_{i}\right)^{\nu_{i}}$, its minimum equation is $F(\lambda) \equiv \prod_{i=1}^{r}\left(\lambda-\alpha_{i}\right)=0$.

Let $e_{i},(i=1,2, \cdots, n)$, and $\phi_{j},(j=1,2, \cdots, r)$, be the principal idempotent elements of $A$ and of $M$, respectively. Then $M=\sum_{i=1}^{n} \beta_{i} e_{i}$ where $\nu_{1}$ of the $\beta$ 's are $\alpha_{1}, \nu_{2}$ are $\alpha_{2}, \cdots$, and $\nu_{r}$ are $\alpha_{r}$. We shall denote the matrix $M$ by the symbol $\left(\beta_{1}, \beta_{2}, \cdots, \beta_{n}\right)_{e i}$. Similarly, we have $M=\sum_{j=1}^{r} \alpha_{j} \phi_{j}$ expressed symbolically by $M=\left(\alpha_{1}, \alpha_{2}, \cdots, \alpha_{r}\right)_{\phi_{j}}$. Then every matrix $\phi_{j}$ is the sum of $\nu_{j}$ of the $e_{i}$, the latter being partial idempotent elements of $M$. For example, if $M=(1,2,1,1,3,2)_{e_{i}}$ $=(1,2,3)_{\phi_{j}}$, we have

$$
\phi_{1}=e_{1}+e_{3}+e_{4}, \quad \phi_{2}=e_{2}+e_{6}, \quad \phi_{3}=e_{5} .
$$

In another paper* the following theorem has been proved:

THEOREM 2. If $\mu$ is the number of distinct latent roots of a matrix $A$, then any polynomial $F(\lambda)$, of degree $t$, with simple zeros, breaks up in $(t !)^{\mu-1}$ ways into a product of $t$ linear factors in the ring $R(A)$.

Since each factorization involves $t$ matrices, all ways of factoring will involve $t \cdot(t !)^{\mu-1}$ matrices, and since $F(\lambda)=0$ has $t^{\mu}$ matric roots equally distributed in the factorizations, it follows that, corresponding to any one of the $t$ matric roots, there will be $t \cdot(t !)^{\mu-1} / t^{\mu}$ or $[(t-1) !]^{\mu-1}$ sets of conjugates. Applying this argument to $F(\lambda) \equiv \prod_{i=1}^{r}\left(\lambda-\alpha_{i}\right)=0$, the minimum equation of $M$, we have the following theorem:

Theorem 3. The matrix $M=\left(\beta_{1}, \beta_{2}, \cdots, \beta_{n}\right)_{e_{i}}=\left(\alpha_{1}, \alpha_{2}, \cdots, \alpha_{r}\right)_{\phi_{j}}$ has, in the ring $R(A),[(r-1) !]^{n-1}$ sets of conjugates with respect to its minimum equation; the matrices of $[(r-1) !]^{r-1}$ of these sets are integral, rational functions of $M$.

ExAMPLE. If $M=(1,2,1,3)_{e_{i}}=(1,2,3)_{\phi_{j}}$, we have the following sets of conjugates in $R(A)$ :

\footnotetext{
* Compositio Mathematica, vol. 6 (1938), pp. 235-238.
} 


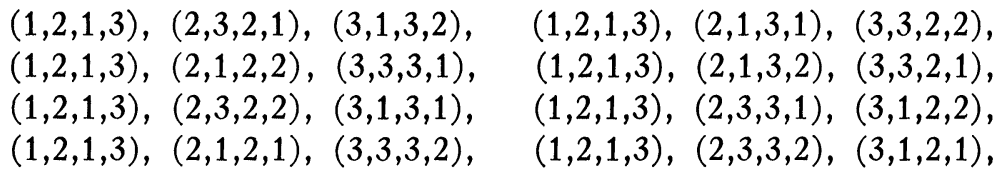

while in $R(M)$ we have

$$
\begin{gathered}
\text { III } \\
(1,2,3),(2,3,1),(3,1,2), \\
(1,2,3), \quad(2,1,2),(3,3,1), \\
(1,2,3), \quad(2,3,2),(3,1,1), \\
(1,2,3),(2,1,1),(3,3,2) .
\end{gathered}
$$

Since $\phi_{1}=e_{1}+e_{3}, \phi_{2}=e_{2}, \phi_{3}=e_{4}$, it is not difficult to identify the sets in group III with those in group I. Thus

$$
\begin{aligned}
& (1,2,3)_{\phi_{j}}=\phi_{1}+2 \phi_{2}+3 \phi_{3}=e_{1}+2 e_{2}+e_{3}+3 e_{4}=(1,2,1,3)_{e_{i}}, \\
& \text { and similarly }(2,3,2)_{\phi_{j}}=(2,3,2,2)_{e_{i}},(3,1,1)_{\phi_{j}}=(3,1,3,1)_{e_{i}} .
\end{aligned}
$$

University of Alabama

\section{TRIPLE SYSTEMS AS RULED QUADRICS*}

W. G. WARNOCK

1. Introduction. If $n$ elements $x_{1}, x_{2}, \cdots, x_{n}$ can be arranged in triples such that each pair $x_{i} x_{j}$ occurs in one and only one triple, the arrangement so formed is a simple triple system. Credit for the first published paper on such systems is given to Kirkman. $\dagger$ Methods of construction, properties, and forms of interpretation of these and more general multiple systems can be found throughout the mathematical literature since that date. $\neq$ In this note I propose to treat the element as a generic line in an ordinary three-space. Likewise, I shall point out some of the group properties which seem worthy of men-

* Presented to the Society, November 28, 1936.

$\dagger$ Kirkman, The Cambridge and Dublin Mathematical Journal, vol. 2 (1847), pp. 192-204.

¥ See A. Emch, Triple and multiple systems, their geometric configurations and groups, Transactions of this Society, vol. 31 (1929), pp. 25-42. An almost complete list of references is given in this paper. A more recent discussion of multiple systems is to be found in an article by R. D. Carmichael, Tactical configurations of rank two, American Journal of Mathematics, vol. 53 (1931), pp. 217-240. 